\title{
Redox neutral and acid-free Minisci C-H alkylation of heteroarenes enabled by dual photoredox/bromide catalysis in micellar solutions
}

\author{
Marilia S. Santos, ${ }^{\text {b† }}$ Martyna Cybularczyk-Cecotka, ${ }^{\text {a† }}$ Burkhard König ${ }^{\mathrm{b}}$ and Maciej Giedyk*a \\ a Institute of Organic Chemistry Polish Academy of Sciences Kasprzaka 44/52, 01-224 Warsaw, Poland \\ b Institute of Organic Chemistry, Faculty of Chemistry and Pharmacy, University of Regensburg, Universitätsstraße 31,93053 \\ Regensburg, Germany. \\ † equal contribution \\ E-mail: maciej.giedyk@icho.edu.pl
}

\begin{abstract}
Microstructured aqueous solutions were employed to engage non-activated alkyl bromides in the visiblelight-promoted $\mathrm{C}-\mathrm{H}$ functionalization of heteroarenes. The reactive carbon-centered alkyl radicals were generated by merging the photoredox approach, bromide anion co-catalysis and spatial pre-aggregation of reacting species in the mixture. The presented methodology allowed obtaining alkylated heteroarenes without stoichiometric radical-promoters, in acid-free conditions and using blue LEDs as the light source.
\end{abstract}

\section{Introduction}

$\mathrm{C}-\mathrm{H}$ alkylation of heteroarenes, known as Minisci reaction, is a well-established synthetic tool for $\mathrm{C}\left(\mathrm{sp}^{2}\right)-\mathrm{C}\left(\mathrm{sp}^{3}\right)$ bond formation. ${ }^{1}$ It enables direct, late-stage modification of aromatic heterocycles, which are omnipresent structural motifs in various natural products, pharmaceuticals and agrochemicals. ${ }^{2}$ The Minisci reaction involves generation of carbon-centered alkyl radicals and their addition to an electron-deficient heteroaromatic ring, which is accompanied by a formal loss of the hydrogen atom.

Various precursors of alkyl radicals have been employed in the Minisci reaction including amino acids, ${ }^{3}$ aldehydes, ${ }^{4-6}$ ketones, ${ }^{7}$ carboxylic acids, ${ }^{8-10}$ alkyltrifluoroborate salts, ${ }^{11}$ pyridinium salts, ${ }^{12,13}$ boronic acids, ${ }^{14,15}$ diazonium salts, ${ }^{16}$ peroxides, ${ }^{17-19}$ alkyl halides ${ }^{20-27}$ etc. Among them, alkyl bromides are of particular synthetic potential, as they are readily available and inexpensive starting materials. However, the cleavage of the relatively strong $\mathrm{C}-\mathrm{Br}$ bond in alkyl bromides, which must occur in the course of the process, presents a major challenge. As a result, only few variants of the Minisci reaction exploiting non-activated alkyl bromides have been reported so far.

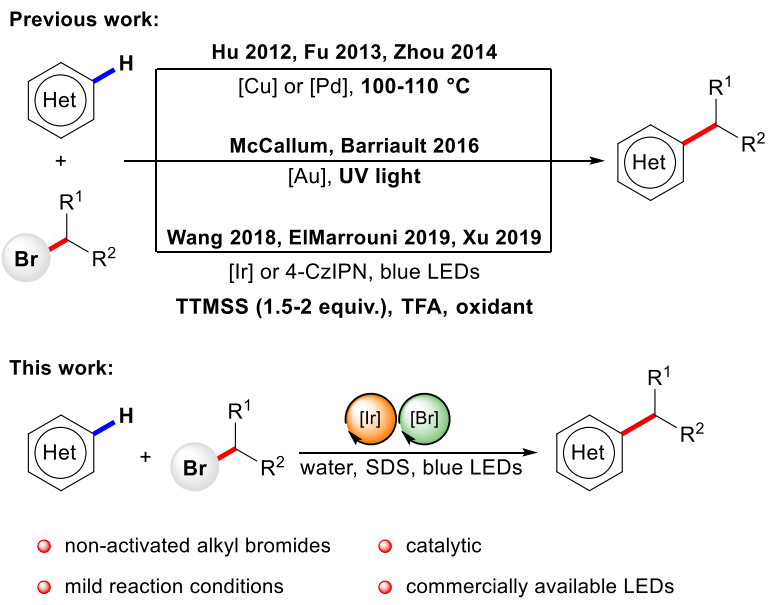

Fig. 1. Strategies for $\mathrm{C}-\mathrm{H}$ alkylation of heteroarenes with non-activated alkyl bromides.

The established strategies to overcome the challenge of $\mathrm{C}-\mathrm{Br}$ bond activation involve the use of high temperatures, strong UV-light irradiation or the addition of stoichiometric amounts of silyl radical-promoters (Fig. 1). Accordingly, in $2012 \mathrm{Hu}$ et al. developed an efficient method for alkylation of benzoxazoles with secondary alkyl halides. ${ }^{22}$ The majority of presented syntheses were realized using alkyl iodides, but few 
examples with bromides have also been reported. The reaction was performed at elevated temperatures, and with the use of copper-based catalyst. One year later, $\mathrm{Fu}$ et al. demonstrated the cross-coupling of non-activated secondary and tertiary alkyl bromides with pyridine $N$-oxides in the presence of palladium catalyst and phosphine ligand. ${ }^{23}$ The method was further developed by Zhou et al., who extended the palladium-catalyzed Minisci reaction on a broad variety of heteroarenes, including indole- and pyridine derivatives. ${ }^{24}$ The photocatalytic alternative towards the activation of alkyl bromides have been presented by McCallum and Barriault. ${ }^{25}$ Using gold complexes as catalysts and UV light as the energy source they performed Minisci reactions with various non-activated bromoalkanes and heteroarenes and supported their studies by the detailed investigations of photophysical and electrochemical properties of the photocatalyst. ${ }^{26}$ Minisci reactions with non-activated alkyl bromides were also investigated by the groups of Wang, EIMarrouni and $\mathrm{Xu}$, who capitalized on the joined action of the photocatalyst, acid, silyl radicalpromoters and visible light irradiation. ${ }^{27-29}$

While these pioneering methods are of unquestionable value, the need for mild, redox-neutral catalytic methods of $\mathrm{C}-\mathrm{H}$ alkylation of heteroarenes with alkyl bromides still remains. In order to address this challenge we resorted to photocatalysis in aqueous structured solutions. We exploited the pre-aggregation of the reacting species and merged it with the autocatalytic role of bromide anions, which were generated in situ from the starting material. ${ }^{30}$ This allowed facilitating the $\mathrm{C}-\mathrm{C}$ coupling of non-activated alkyl bromides with heteroarenes without stoichiometric radical-promoters, in acid-free conditions and with commercial blue LEDs as the light source.

\section{Results and discussion}

The redox potential of typical photocatalysts in their excited state, including strongly reducing Ir-species, precludes the direct single-electron-transfer (SET) to alkyl bromides $(-2.29 \mathrm{~V}$ vs. SCE for 1 -bromooctane $)^{31}$. However, catalytic species of a much higher reducing power can be generated via the reductive quenching of the catalyst followed by the subsequent excitation with a second visible-lightphoton. ${ }^{30,32-34}$ Although the typically used reductive quenchers include tertiary amines, Hantzsch esters, alcohols, ascorbate anions etc., ${ }^{35}$ it has recently been shown that the efficient quenching of excited Ir-complexes can also be achieved using simple halide anions, leading to $\operatorname{Ir}(\mathrm{II})$-species and halide radicals. ${ }^{36-40}$ We decided to test, if $\mathrm{Br}^{-}$anions, which are released upon the single-electron-reduction and fragmentation of alkyl bromides, can be recycled and used as mediators in the Minisci reaction - quench the excited Ir(III)-photocatalyst and thus promote the generation of alkyl radicals.

Unique properties of structured solutions of surfactants, combined with operationally simple preparation, render them advantageous media for chemical reactions such as biocatalysis, ${ }^{41}$ polymerizations, ${ }^{42}$ transition-metal catalyzed cross-coupling reactions, ${ }^{43}$ and organocatalytic transformations ${ }^{44}$. Recent reports show that they may also play a vital role in photocatalysis. ${ }^{30,45-48}$ From the viewpoint of the designed Minisci reaction, structured aqueous solution could provide the necessary pre-association of the starting materials and the photocatalyst, improve the kinetics of the reaction and thus eliminate the harsh reaction conditions or stoichiometric additives, including radical promotors and acids.

In order to test the working hypothesis, we subjected bromocyclohexane (2a) to the reaction with lepidine (1a) in the presence of $\operatorname{Ir}(\mathrm{dtbby})(\mathrm{ppy})_{2} \mathrm{PF}_{6}(\mathbf{4})$ as photocatalyst, in aqueous solution of surfactant and under irradiation with blue LEDs (Table 1). We were pleased to see that the reaction proceeded and the desired coupling product $\mathbf{3 a}$ was formed in $31 \%$ yield (entry 1 ). The reaction parameters were then optimized with respect to the surfactant, photocatalyst, co-catalyst, time, as well as the ratio and concentration of reagents (for full optimization studies see $\mathrm{SI}$ ). The addition of the catalytic amount of $\mathrm{NaBr}$ facilitated the process and increased the yield of compound $3 \mathrm{a}$ to $47 \%$ (entry 2). Further screening established $\mathrm{CBr}_{4}$ as a co-catalyst of choice (entries 2-5). The applied conditions, which were called Procedure A, afforded the full conversion of lepidine 1a and the desired product $\mathbf{3 a}$ in $91 \%$ yield. The alkylation occurred selectively at position C2. Although the optimal reaction conditions involved $20 \mathrm{~mol} \%$ of $\mathrm{CBr}_{4}$ and 42 hours of 
irradiation, the efficient formation of the product 3a was observed already after shorter reaction time (52\% after 18 h, 78\% after 24 h) or using lower co-catalyst loading (5 mol\%) (entries 6 and 7, respectively). Among various tested photocatalysts, the highest activity was achieved using $\operatorname{Ir}(\mathrm{dtbbpy})(\mathrm{ppy})_{2} \mathrm{PF}_{6}(\mathbf{4})$. Other mediators proved inefficient (entries 8, 10-12), or provided the product $\mathbf{3 a}$ in low yield (entry 9). Having catalyst and co-catalyst selected, we evaluated the influence of popular and readily available surfactants. The superior performance of sulfate-based surfactants: sodium dodecyl sulfate (SDS) and sodium lauryl oligoethylene glycol sulfate (SLES) was observed (entries 1 - 13), which is in agreement with previous reports. ${ }^{30}$ The satisfactory $41 \%$ yield of the desired product 3 a was also detected when zwitterionic surfactant SB3-14 was used (entry 15). The application of anionic potassium dodecanate, cationic dodecyltrimethylammonium chloride (DTAC) or non-ionic Triton X-100 led to less efficient product 3a formation (entries 14, 16, 17).

Table 1. Optimization studies ${ }^{a}$

\begin{tabular}{cccccc} 
& & & & \\
\hline
\end{tabular}

aReaction conditions: lepidine $1 \mathbf{a}(0.1 \mathrm{mmol})$, bromocyclohexane $2 \mathbf{a}(0.2 \mathrm{mmol})$, surfactant $(0.25 \mathrm{mmol})$, co-catalyst $(20 \mathrm{~mol} \%)$, photocatalyst $(3 \mathrm{~mol} \%)$, water $(5 \mathrm{~mL}), 40^{\circ} \mathrm{C}, 451 \mathrm{~nm}, 42 \mathrm{~h}$. ${ }^{b}$ Yields were calculated using GC analysis. $n$-Dodecane was used as

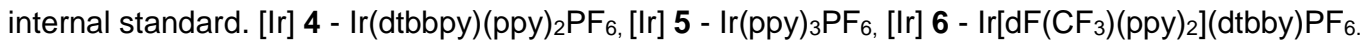

With the reaction conditions established, we next investigated the scope of the developed transformation (Table 2). In general, secondary bromides $\mathbf{2 a - 2 d}$ provided higher yields of the desired products than primary ones $\mathbf{2 e - 2}$, which reflects higher thermodynamic stability of the intermediate radicals. However, the precursors $\mathbf{2 m - 0}$ of even more stabilized benzyl, tertiary or a-carbonyl radicals, proved unsuitable, presumably due to the competing oxidation to carbocations and hydrolysis, which led to respective alcohols. Several functional groups in the bromide moiety showed good compatibility with our procedure such as free hydroxyl group (3h), primary (3k) and secondary amides (3l), chlorides $(\mathbf{3 g})$ or $\mathrm{CF}_{3}$ function (3j). Additionally, the product $\mathbf{3 i}$ possessing a terminal double bond was also isolated. Alkyl bromides $\mathbf{2 p}$ decorated with acetal groups proved unstable under the reaction conditions. Evaluation of the aromatic coupling partners showed that the reaction is compatible not only with simple heterocycles such as lepidine 1a, phenantridine 1b and quinoline 1c, but also derivatives, which contain ester or cyano substituents. 4-Phenylpyridine $1 \mathbf{d}$ gave a mixture of mono- and disubstituted products $10 \mathbf{d}$ and di-10d, both of which could be selectively isolated ( $38 \%$ and $40 \%$ respectively). In the case of nicotinonitrile 1e and methylnicotinate 1f, the increase in the amount of alkyl bromide (from 3 to 5 equiv.) led to selective formation of tri-substituted products $\mathbf{1 0 e}$ and tri-10f in very good yields. Alternatively, by keeping the 
standard reaction conditions, the di-substituted compound $\mathbf{1 0 f}$ was obtained as the main product. The two alkyl groups were appended selectively at positions $C 4$ and C6, as indicated by 2D NMR studies (see SI). The observed limitations on the side of the heteroaromatic partner included the compounds with blocked position C2, heterocycles possessing aldehyde or ketone groups or substrates with halogen substituents, for which the undesired dehalogenation reactions prevailed.

Table 2. Scope of the reaction ${ }^{a}$

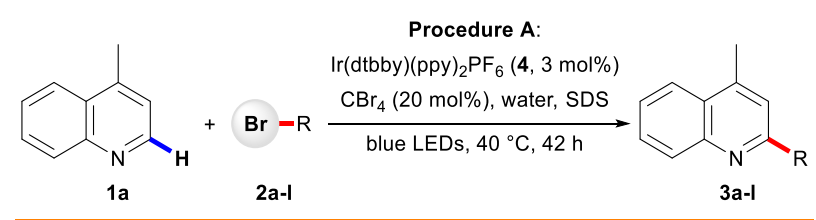

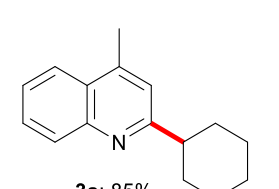

3a: $85 \%$

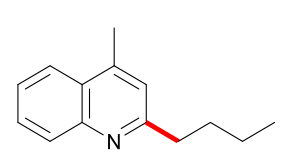

3e: $48 \%$

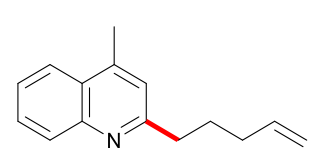

3i: $17 \%$
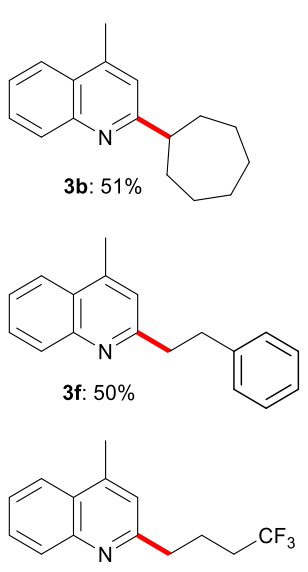

3 j: $50 \%$

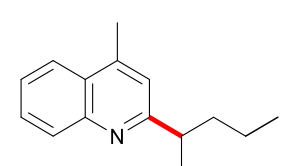

3c: $56 \%$

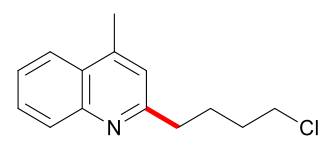

3g: $57 \%$

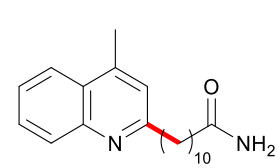

3k: $44 \%$

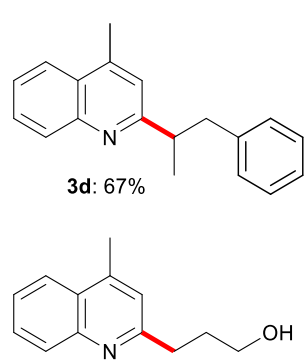

3h: $70 \%$

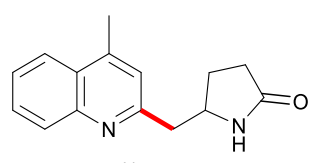

3I: $28 \%$ unsuccessful

substrates:

$\mathrm{Ph} \mathrm{Br}$

$2 \mathrm{~m}$

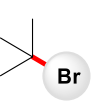

2n

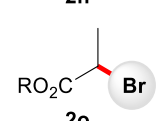

20

$\overbrace{O R}^{\mathrm{Br}}$

2p
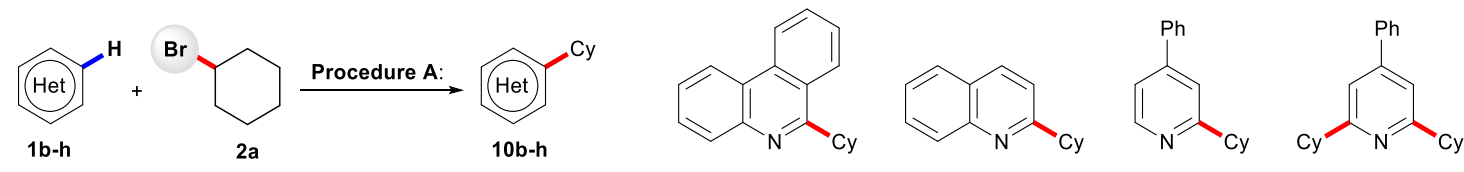

10b: $55 \%$

10c: $42 \%^{b}$

10d: $38 \%$

di-10d: $40 \%$<smiles>N#Cc1c(Cl)cc(Cl)nc1Cl</smiles>

10e: $85 \%^{c}$<smiles>CC(=O)c1cnc(Cl)cc1Cl</smiles>

10f: $49 \%$

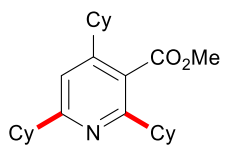

tri-10f: $75 \%^{c}$

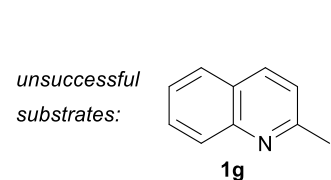

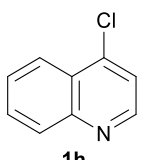

$1 \mathrm{~h}$

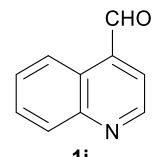

${ }^{a}$ Average isolated yield obtained from two separate reactions are given. ${ }^{b}$ Isolated in a 3:1 mixture with 2,4-dicyclohexylquinoline. ${ }^{c}$ Reactions were carried out for $20 \mathrm{~h}$ with 5 equiv. of alkyl bromide.

In order to gain more insights into the studied reaction, a series of mechanistic experiments was conducted. The control reactions showed that light, the photocatalyst and the surfactant are all essential for this Minisci protocol (Table 3, entries 2, 3 and 4). Only small portions of the heteroarene convert in the absence of the alkyl bromide as the second reagent (entry 5). Furthermore, the addition of $\mathrm{CBr}_{4}$ facilitates the developed reaction and lower yield (31\%) of the model product 3a was obtained in its absence (entry 6 ). To evaluate the impact of the micellar solution as the reaction environment, the control reaction in acetonitrile was performed. Although a clear solution indicated good solubility of all of the reaction components, the formation of product 3a was not detected (entry 7). No desired reaction was also observed when 2,2,6,6-tetramethylpiperidinyloxyl (TEMPO) was employed under the optimized conditions, which indicates the presence of radical intermediates in the reaction mechanism (entry 8). 
Table 3. Control experiments

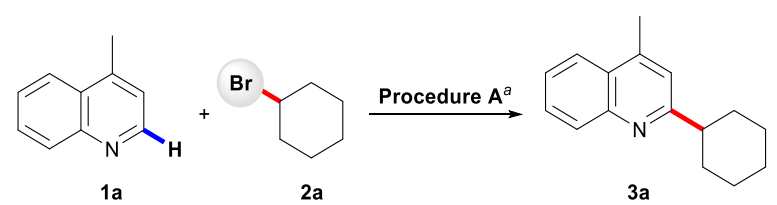

\begin{tabular}{cccc}
\hline No. & $\begin{array}{c}\text { Variation from optimized } \\
\text { conditions }^{a}\end{array}$ & $\begin{array}{c}\text { Conversion } \\
\mathbf{1 a}^{b}[\%]\end{array}$ & $\begin{array}{c}\text { Yield } \mathbf{3} \mathbf{a}^{b} \\
{[\%]}\end{array}$ \\
\hline 1 & - & 100 & 91 \\
2 & no photocatalyst $\mathbf{4}$ & 23 & 0 \\
3 & no light & 20 & 0 \\
4 & no SDS & 22 & 1 \\
5 & no bromocyclohexane (2a) & 13 & 0 \\
6 & no CBr & \\
7 & MeCN instead of aqueous SDS $_{4}$ & 37 & 31 \\
8 & with the addition of TEMPO & \\
\hline
\end{tabular}

aOptimized conditions: lepidine (1a, $0.1 \mathrm{mmol})$, bromocyclohexane (2a, $0.2 \mathrm{mmol})$, SDS (0.25 mmol), CBr 4 (20 mol\%), $\operatorname{Ir}(\mathrm{dtbbpy})(\mathrm{ppy})_{2} \mathrm{PF}_{6}(\mathbf{4}, 3 \mathrm{~mol} \%)$, water $(5 \mathrm{~mL}), 40{ }^{\circ} \mathrm{C}, 451 \mathrm{~nm}, 42 \mathrm{~h} .{ }^{\mathrm{b} C}$ Conversion and yield were calculated using $\mathrm{GC}$ analysis. $n$-Dodecane was used as internal standard; 22 equiv. of TEMPO were added to the reaction mixture.

To further examine the mechanistic pathway, we conducted a radical-clock experiment starting from 5-bromo-1-hexene (2r) (Fig. 2a). The presence of the cyclopentane ring in the main product 3r suggests the formation of carbon-centered radicals, which undergo fast 5-exo-trig cyclization and subsequent addition to the heteroarene 1a. The Stern-Volmer fluorescence quenching experiment was performed, to examine the interactions of the photocatalyst with other reaction components (Fig. $2 b) .{ }^{49}$ It showed that the excited state of $\operatorname{Ir}(\mathrm{dtbbpy})(\mathrm{ppy})_{2} \mathrm{PF}_{4}$ (4) is quenched effectively by $\mathrm{CBr}_{4}$, while only low quenching efficiency was observed for the lepidine (1a) or the alkyl bromide 2a. These results are congruent with the high redox potential of alkyl bromides and nitrogen heterocycles. ${ }^{50}$ The reaction progress was monitored over time, showing the increasing rate of the process within the first 10 hours of irradiation (Fig. 2c). It supports the concept of the autocatalytic role of bromide anions. Their accumulation in the reaction mixture increases the quenching efficiency and provides higher concentration of the reduced form of the photocatalyst 4. Moreover, a significant change in the $\mathrm{pH}$ of the reaction mixture, from basic $(\mathrm{pH}=10)$ to acidic $(\mathrm{pH}=3)$ was observed over time.

a)

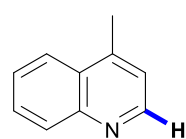

$1 \mathrm{a}$

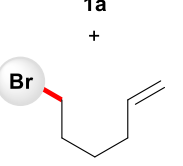

$2 r$

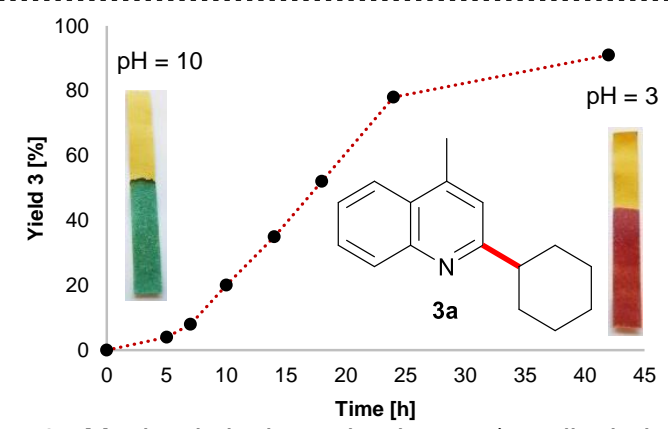

b)
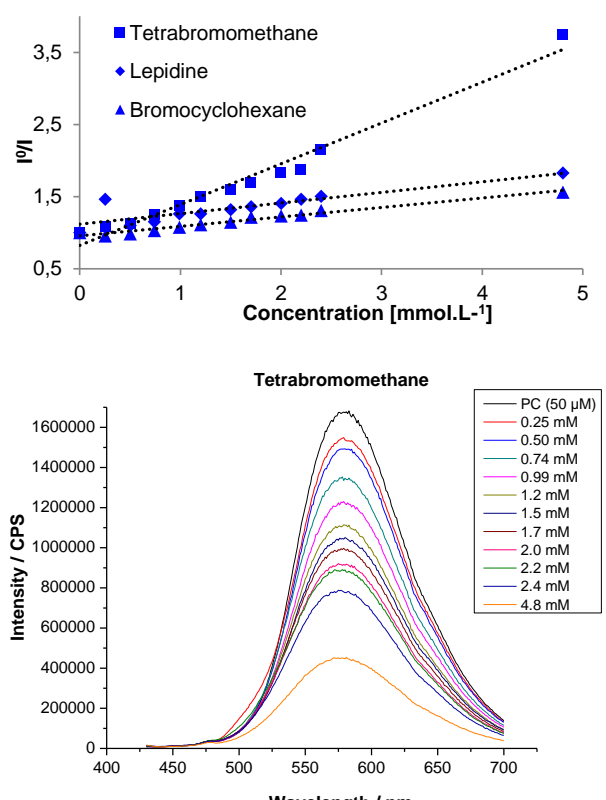

Fig. 2. Mechanistic investigations: a) radical-clock experiment with 6-bromo-1-hexene (2r); b) Stern-Volmer fluorescence quenching of $\operatorname{Ir}(\mathrm{dtbbpy})(\mathrm{ppy})_{2}(\mathrm{c}=50 \mu \mathrm{M})$ in aqueous SDS; $\left.\mathrm{c}\right)$ Kinetic studies of the model reaction and the change in the pH of the reaction progress. 
In accordance with these results, as well as the optimization studies, we propose a mechanism of the developed Minisci reaction (Fig. 3). It has been firmly established that the excited [Ir] 4 photocatalyst $\left(E_{1 / 2}\right.$ $=-1.51 \mathrm{~V}$ vs. SCE in acetonitrile $)^{51}$ undergoes reductive quenching by $\mathrm{Br}^{-}$anions $\left(\mathrm{E}_{1 / 2}=+0.80 \mathrm{~V} \mathrm{vs}\right.$. SCE in DME). ${ }^{36-39}$ Consequently, a bromine radical and the reduced $\operatorname{Ir}(\mathrm{II})$-complex are generated. The latter species can undergo consecutive absorption of a second photon, resulting in the formation of a strongly reducing form of the iridium-complex ${ }^{30,32}$ or a solvated electron. ${ }^{34}$ SET to alkyl bromide $\mathbf{A}$ followed by fragmentation affords alkyl radical $\mathbf{B}$ and a bromide anion, which participates in subsequent catalytic cycles. An addition of alkyl radical $\mathbf{B}$ to pyridinium salt $\mathbf{C}$ provides the radical cation $\mathbf{D}$, able to undergo hydrogen-atom-transfer (HAT) with an electrophilic bromine radical. As a result, the protonated form $\mathbf{E}$ of the final product is produced. Additionally, the contribution of radical propagation through the interaction of radical cation $\mathbf{D}$ with alkyl bromide $\mathbf{A}$ should also be considered.

Detailed mechanistic studies on the role of $\mathrm{CBr}_{4}$ co-catalyst are ongoing, but preliminary results suggest that, through the photosensitized hydrolysis of $\mathrm{CBr}_{4}$, it may provide the starting concentration of bromide anions at the early stage of the process. Although the light-induced reactivity of this compound is usually associated with mesolytic bond cleavage, ${ }^{52-55}$ or homolytic dissociation to $\mathrm{CBr}_{3}$ and $\mathrm{Br}$ radicals, ${ }^{56,57}$ it has been shown that in the aqueous conditions the photoinduced hydrolytic pathway to $\mathrm{HBr}$ prevails. ${ }^{58}$ Alternatively, the reduction of $\mathrm{CBr}_{4}$ by excited $\operatorname{Ir}(\mathrm{III})^{*}$-photocatalyst can be considered, leading to $\mathrm{Br}^{-}$, the $\mathrm{CBr}_{3}$ radical and $\operatorname{Ir}(\mathrm{IV})$-complex. The last two species may undergo SET to recover $\operatorname{Ir}(\mathrm{III})$ and produce $\mathrm{CBr}_{3}$ cation, which reacts with water to give the tribromomethanol and a proton. Finally, tetrabromomethane may contribute to the overall reaction outcome through yet another catalytic mode. Due to the halogen bonding with bromide anions, ${ }^{59,60}$ it may decrease their hydrophilic character, slow down the migration to the water bulk and, consequently, render $\mathrm{Br}^{-}$more accessible to the excited $\operatorname{Ir}(\mathrm{III})^{*}$ photocatalyst.

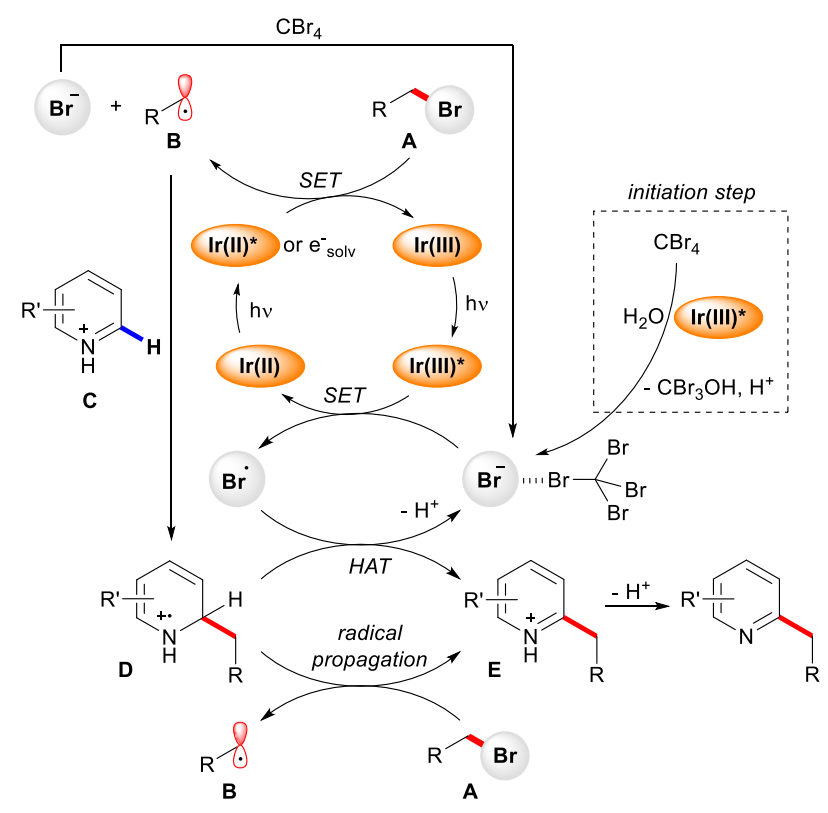

Fig. 3. Proposed mechanistic pathway.

To further examine the decisive role of the solution structuring, in particular the postulated pre-arrangement of bromide anions, we investigated the reaction in the presence of the cationic surfactant cetyltrimethylammonium bromide (CTAB) and catalytic amount of $\mathrm{NaBr}$ instead of $\mathrm{CBr}_{4}$. The positively charged head of the surfactant would retain the bromide counter-anion through ion-pairing interactions and keep it in a close distance to the interface, thus favoring the interaction with the photocatalyst. We were pleased to find, that, under this condition, which were called Procedure B, the compound $3 \mathbf{3}$ a was obtained in $88 \%$ yield (Table 4). Moreover, the use of $C T A B$ as a sole source of bromide anions, without external $\mathrm{NaBr}$ added, also afforded the desired product $3 \mathbf{a}$ in good yield (66\%). Finally, we demonstrated that the Procedure $B$ can be successfully 
implemented to obtain alkylated heterocycles $\mathbf{3 b}, \mathbf{3 h}$ and $\mathbf{1 0 d}$ from other aliphatic bromides and heteroarenes in good efficiency.

Table 4. The C-H alkylation of heteroarenes using cationic surfactant with bromide counter ion.

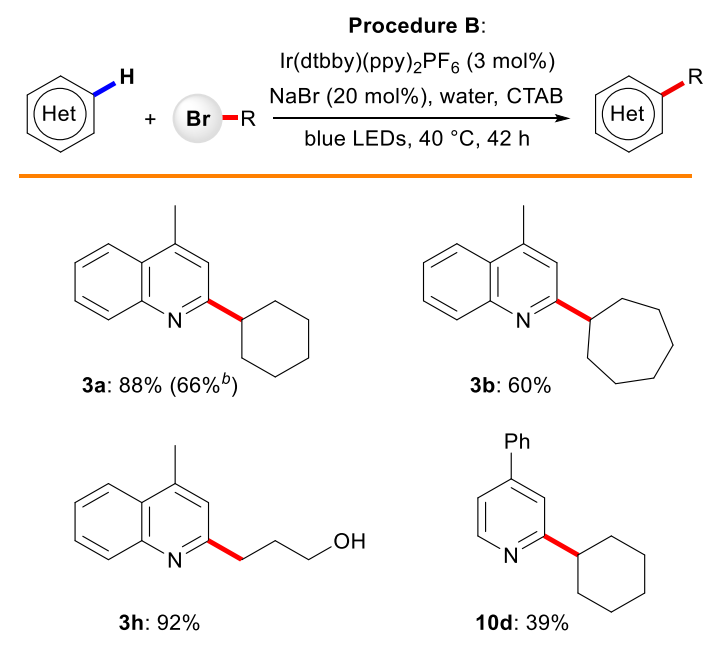

${ }^{a}$ Yields were calculated using NMR analysis with 1,3,5-trimethoxybenzene as an internal standard. ${ }^{b}$ The reaction was performed in the absence of $\mathrm{NaBr}$.

\section{Conclusions}

In summary, we have developed a new photocatalytic procedure for Minisci-type coupling of heteroarenes with various alkyl bromides, which exploits the combination of photoredox catalysis with bromide anion catalysis. With the use of micellar solution as the reaction media, it is possible to carry out the reaction in mild, aqueous conditions, with no need for external oxidant or stoichiometric radical promoter. The coupling products were obtained in the absence of equimolar amounts of acid, a requirement for standard Minisci protocols. The external additives are simple and cost-efficient and they were used in catalytic amounts. The obtained optimization data and mechanistic experiments highlight the critical importance of microstructuring and pre-organization of the components in the reaction mixture.

\section{Conflicts of interest}

There are no conflicts to declare

\section{Acknowledgements}

We gratefully acknowledge funding from the National Science Centre, Poland (SONATA 2018/31/D/ST5/00306), Fundação de Amparo à Pesquisa do Estado de São Paulo - FAPESP (2018/20956-5 and 2017/03120-8) and the German Science Foundation (DFG, KO 1537/18-1) for the financial support.

\section{TOC}

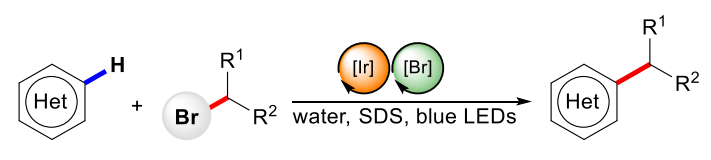

$$
\begin{aligned}
& \text { - non-activated alkyl bromides } 0 \text { catalytic } \\
& \text { - mild reaction conditions } \quad \text { commercially available LEDs }
\end{aligned}
$$




\section{Notes and references}

1. R. S. J. Proctor and R. J. Phipps, Angew. Chemie Int. Ed., 2019, 2-36.

2. K. C. Majumdar and S. K. Chattopadhyay, Eds., Heterocycles in Natural Product Synthesis, Wiley-VCH Verlag GmbH \& Co. KGaA, Weinheim, Germany, 2011.

3. D. N. Mai and R. D. Baxter, Org. Lett., 2016, 18, 3738-3741.

4. K. Matcha and A. P. Antonchick, Angew. Chemie - Int. Ed., 2013, 52, 2082-2086.

5. Y. Siddaraju, M. Lamani and K. R. Prabhu, J. Org. Chem., 2014, 79, 3856-3865.

6. Z. Wang, X. Ji, J. Zhao and H. Huang, Green Chem., 2019, 21, 5512-5516.

7. P. Liu, W. Liu and C. J. Li, J. Am. Chem. Soc., 2017, 139, 14315-14321.

8. W.-F. Tian, C.-H. Hu, K.-H. He, X.-Y. He and Y. Li, Org. Lett., 2019, 21, 6930-6935.

9. F. Minisci, R. Bernardi, F. Bertini, R. Galli and M. Perchinummo, Tetrahedron, 1971, 27, 3575-3579.

10. J. Kan, S. Huang, J. Lin, M. Zhang and W. Su, Angew. Chemie - Int. Ed., 2015, 54, 2199-2203.

11. G. A. Molander, V. Colombel and V. A. Braz, Org. Lett., 2011, 13, 1852-1855.

12. F. J. R. Klauck, M. J. James and F. Glorius, Angew. Chemie - Int. Ed., 2017, 56, 12336-12339.

13. X. Ma and S. B. Herzon, J. Am. Chem. Soc., 2016, 138, 8718-8721.

14. G. X. Li, C. A. Morales-Rivera, Y. Wang, F. Gao, G. He, P. Liu and G. Chen, Chem. Sci., 2016, 7, 6407-6412.

15. I. B. Seiple, S. Su, R. A. Rodriguez, R. Gianatassio, Y. Fujiwara, A. L. Sobel and P. S. Baran, J. Am. Chem. Soc., 2010, 132, 1319413196.

16. D. Xue, Z. H. Jia, C. J. Zhao, Y. Y. Zhang, C. Wang and J. Xiao, Chem. - A Eur. J., 2014, 20, 2960-2965.

17. D. A. DiRocco, K. Dykstra, S. Krska, P. Vachal, D. V. Conway and M. Tudge, Angew. Chemie - Int. Ed., 2014, 53, 4802-4806.

18. F. Minisci, C. Giordano, E. Vismara, S. Levi and V. Tortelli, J. Am. Chem. Soc., 1984, 106, 7146-7150.

19. F. Minisci, E. Vismara, F. Fontana, G. Morini, M. Serravalle and C. Giordano, J. Org. Chem., 1986, 51, 4411-4416.

20. N. B. Bissonnette, M. J. Boyd, G. D. May, S. Giroux and P. Nuhant, J. Org. Chem., 2018, 83, 10933-10940.

21. P. Nuhant, M. S. Oderinde, J. Genovino, A. Juneau, Y. Gagné, C. Allais, G. M. Chinigo, C. Choi, N. W. Sach, L. Bernier, Y. M. Fobian, M. W. Bundesmann, B. Khunte, M. Frenette and O. O. Fadeyi, Angew. Chemie - Int. Ed., 2017, 56, 15309-15313.

22. P. Ren, I. Salihu, R. Scopelliti and X. Hu, Org. Lett., 2012, 14, 1748-1751.

23. B. Xiao, Z. J. Liu, L. Liu and Y. Fu, J. Am. Chem. Soc., 2013, 135, 616-619.

24. X. Wu, J. W. T. See, K. Xu, H. Hirao, J. Roger, J.-C. Hierso and J. S. Zhou, Angew. Chemie, 2014, 126, 13791-13795.

25. T. McCallum and L. Barriault, Chem. Sci., 2016, 7, 4754-4758.

26. C. D. McTiernan, M. Morin, T. McCallum, J. C. Scaiano and L. Barriault, Catal. Sci. Technol., 2016, 6, $201-207$.

27. J. Dong, X. Lyu, Z. Wang, X. Wang, H. Song, Y. Liu and Q. Wang, Chem. Sci., 2019, 10, 976-982.

28. J. J. Perkins, J. W. Schubert, E. C. Streckfuss, J. Balsells and A. EIMarrouni, European J. Org. Chem., 2019, 4, 2-10.

29. R. Chang, J. Fang, J.-Q. Chen, D. Liu, G.-Q. Xu and P.-F. Xu, ACS Omega, 2019, 4, 14021-14031.

30. M. Giedyk, R. Narobe, S. Weiß, D. Touraud, W. Kunz and B. König, Nat. Catal., 2020, 3, 40-47.

31. P. W. Jennings, D. G. Pillsbury, J. L. Hall and V. T. Brice, J. Org. Chem., 1976, 41, 719-722.

32. T. U. Connell, C. L. Fraser, M. L. Czyz, Z. M. Smith, D. J. Hayne, E. H. Doeven, J. Agugiaro, D. J. D. Wilson, J. L. Adcock, A. D. Scully, D. E. Gómez, N. W. Barnett, A. Polyzos and P. S. Francis, J. Am. Chem. Soc., 2019, 141, 17646-17658.

33. I. Ghosh, T. Ghosh, J. I. Bardagi and B. König, Science (80-. )., 2014, 346, 725 LP - 728.

34. F. Glaser, C. Kerzig and O. S. Wenger, Angew. Chemie, , DOI:10.1002/ange.201915762.

35. D. Petzold, M. Giedyk, A. Chatterjee and B. König, European J. Org. Chem., 2020, 2020, 1193-1244.

36. P. Zhang, C. C. Le and D. W. C. MacMillan, J. Am. Chem. Soc., 2016, 138, 8084-8087.

37. T. Kawasaki, N. Ishida and M. Murakami, J. Am. Chem. Soc., 2020, 142, 3366-3370.

38. Z. Wang, X. Ji, T. Han, G.-J. Deng and H. Huang, Adv. Synth. Catal., 2019, 361, 5643-5647.

39. Z. Wang, Q. Liu, X. Ji, G.-J. Deng and H. Huang, ACS Catal., 2020, 10, 154-159.

40. M. Zidan, A. O. Morris, T. McCallum and L. Barriault, European J. Org. Chem., , DOI:10.1002/ejoc.201900786.

41. M. Cortes-Clerget, N. Akporji, J. Zhou, F. Gao, P. Guo, M. Parmentier, F. Gallou, J. Y. Berthon and B. H. Lipshutz, Nat. Commun., 2019, 10, 1-10.

42. I. Capek and T. Kocsisova, Des. Monomers Polym., 2011, 14, 327-345.

43. S. Handa, B. Jin, P. P. Bora, Y. Wang, X. Zhang, F. Gallou, J. Reilly and B. H. Lipshutz, ACS Catal., 2019, 9, $2423-2431$.

44. L. Baxová, R. Cibulka and F. Hampl, J. Mol. Catal. A Chem., 2007, 277, 53-60.

45. M. Bu, G. Lu, J. Jiang and C. Cai, Catal. Sci. Technol., 2018, 8, 3728-3732.

46. T. Kohlmann, C. Kerzig and M. Goez, Chem. - A Eur. J., 2019, 25, 9991-9996.

47. C. Kerzig and M. Goez, Chem. Sci., 2016, 7, 3862-3868.

48. R. Naumann, F. Lehmann and M. Goez, Angew. Chemie Int. Ed., 2018, 57, 1078-1081.

49. M. H. Gehlen, J. Photochem. Photobiol. C Photochem. Rev., 2020, 42, 100338.

50. H. G. Roth, N. A. Romero and D. A. Nicewicz, Synlett, 2016, 27, 714-723.

51. C. K. Prier, D. A. Rankic and D. W. C. MacMillan, Chem. Rev., 2013, 113, 5322-5363.

52. M. J. W. Taylor, W. T. Eckenhoff and T. Pintauer, Dalt. Trans., 2010, 39, 11475-11482.

53. M. Pirtsch, S. Paria, T. Matsuno, H. Isobe and O. Reiser, Chem. - A Eur. J., 2012, 18, 7336-7340.

54. M. N. C. Balili and T. Pintauer, Dalt. Trans., 2011, 40, 3060-3066.

55. T. Hou, P. Lu and P. Li, Tetrahedron Lett., 2016, 57, 2273-2276.

56. Q. Kong, M. Wulff, J. H. Lee, S. Bratos and H. Ihee, J. Am. Chem. Soc., 2007, 129, 13584-13591.

57. S. Tripathi, S. N. Singh and L. D. S. Yadav, RSC Adv., 2016, 6, 14547-14551.

58. C. Zhao, X. Lin, W. M. Kwok, X. Guan, Y. Du, D. Wang, K. F. Hung and D. L. Phillips, Chem. - A Eur. J., 2005, 11, $1093-1108$.

59. G. Cavallo, P. Metrangolo, R. Milani, T. Pilati, A. Priimagi, G. Resnati and G. Terraneo, Chem. Rev., 2016, 116, $2478-2601$.

60. T. Brinck and A. N. Borrfors, J. Mol. Model., , DOI:10.1007/s00894-019-4014-7. 